\title{
Sharps injuries among health care workers in Al Ahsa region, Saudi Arabia
}

\author{
Hanan Ahmed Al Shaikh, Maher Mohammad Al Mahdi, Balaji Rama Naik \\ Ministry of Health, Al Ahsa, Saudi Arabia \\ doi: $10.3396 /$ ijic.v15i4.017.19
}

\begin{abstract}
Data on needle stick injuries and other sharps injuries were collected and analysed for a period of three years from 2016 to 2018 to see the probable risk factors including, for example, type of device, location of injuries, work site, and job category. Nurses were found to be more prone for injuries, with hollow bore needles reported as the most common device involved. Housekeepers formed a major portion of staff with reported injuries and with source unknown. There are gaps in collecting data and quality of data. Focused training for high risk categories, with customized materials suitable to the staff, are recommended to prevent the injuries.
\end{abstract}

Keywords: Sharps injuries, healthcare workers, Saudi Arabia

\section{Corresponding Author}

Balaji Rama Naik

Infection Control Specialist, Infection Prevention and Control Administration, Gate number 8, Directorate of Health Affairs, Ministry of Health, Al Ahsa, Saudi Arabia.

Email: drbalajinaik@gmail.com 


\section{Introduction}

Sharps injuries among health care workers (HCWs) are preventable occupational health hazards. ${ }^{1}$ The consequences of injuries caused by needles and other sharps can be disastrous due to the potential of contracting serious and often fatal blood borne diseases. Reportedly more than 20 different types of blood borne pathogens, such as hepatitis $B$, hepatitis $C$, and human immunodeficiency virus (HIV), can be acquired by the person exposed to needlestick injuries (NSIs) or sharps injures. ${ }^{2}$ The exact magnitude of this problem is unknown. Globally an estimated 6\% of 35 million HCWs are exposed every year. ${ }^{3}$ Data available are from studies which have in-built bias, as most of the studies are based on self-reporting. Data from the "Exposure Prevention Information Network" (EPINet) show that nearly 30 needle stick injuries per 100 beds per year occur in USA. ${ }^{4}$ Limited information is available on the magnitude of the problem in the Gulf region. All the hospitals in the Ministry of Health hospitals in Al Ahsa region capture the data on sharps injuries in EPINet. The aim of this study was to collect information for the years 2016 to 2018 about the incidence of injuries in hospitals of Al Ahsa, Saudi Arabia, with the following objectives: (a) to assess the frequency of sharps injuries in hospitals; (b) to assess the various risk factors associated with sharps injuries such as job category, procedure, site etc.; (c) to look for patterns or seasonal variations.

\section{Methods}

This was a cross sectional study based on the data collected over three years from 2016 to 2018. The study was done in Al Ahsa governorate with a population above 945,631 comprising of major cities such as Al-Hofuf, Al-Mubarraz, Al-Oyoon, Al-Omran and others. It is one of the largest governorates in Saudi Arabia's Eastern Province. The study included all the 11 public sector hospitals and one private sector hospital implementing the EPINet program to report NSIs and sharps injuries. As per the national guidelines, any HCW exposed to occupational injury has to report to the employee health clinic (EHC) or emergency department. The details of the injury and further management are noted in prescribed format which are later entered into the EPINet software system. The exposed HCWs are managed based on the type of exposure in line with the national guidelines.
The study population included all HCWs of the hospitals implementing the EPINet program. The data of NSls and sharps injuries are entered into the EPINet program by the EHC staff on a real-time basis. A monthly report is generated by the hospitals, based on the entries made in the EPINet program. These monthly reports were collected from all the hospitals which were part of this study and checked for completeness and validated by the authors. Excel (Microsoft, Redmond, WA) was used to create a master database which was then used for analysis. All variables have been described as proportions, and the differences between groups have been compared for statistical significance using the chi square test or Fishers exact test, as applicable. The $p$ value was set at 0.05 to consider as statistically significant.

\section{Ethics approval}

The study protocol was reviewed and approved by The Research Committee of King Fahad Hospital Hofuf, Directorate of Health Affairs, Ministry of Health; Al Ahsa. Saudi Arabia. The research proposal approval reference number was KFHH RCA NO: 04/ 2019.

\section{Results:}

Nurses were the most frequently exposed HCWs among all, with $48 \%$ of total reported injuries (Table I). Attending doctors, housekeepers and resident doctors constituted 11\%, $10 \%$ and $9 \%$ of cases respectively. Compared to the three shifts, morning shift was found to be the most common time when incidents of sharps injuries occurred. Location-wise the highest proportion of injuries was recorded in patient room which constituted $30 \%$ of incident cases, followed by the emergency department and operating room which contributed $19 \%$ each. In the majority of the cases (77\%), the injured worker was the original user of the sharp item. The source patient was known in $73 \%$ of the total 262 reported injuries.

As seen in Table II, more than $50 \%$ of injuries were during the procedure, with $40 \%$ of them occurring during the use of the item and about $12 \%$ occurred between the steps of a multi-step procedure. Most of the injuries took place during activities such as injection (22\%), drawing blood (15\%) and suturing (13\%). Hollow bore needles were found to be the device involved in $69 \%$ of cases compared to surgical/ 
Table I. Characteristics of health care workers exposed to needle stick injuries or sharps injuries, 20162018, Al Ahsa, Saudi Arabia ( $n=262$ )

\begin{tabular}{lr} 
Characteristic & Number (\%) \\
\hline Job category of the exposed worker & $126(48)$ \\
\hline Nurse & $30(11)$ \\
\hline Attending doctor & $26(10)$ \\
\hline Housekeeper & $23(9)$ \\
\hline Resident doctor & $13(5)$ \\
\hline Nursing student & $44(17)$ \\
\hline Others & \\
\hline & \\
\hline Duty shift when the injury occurred & $178(69)$ \\
\hline Morning & $29(11)$ \\
\hline Evening & $51(20)$ \\
\hline Night
\end{tabular}

Work location where injury occurred

\begin{tabular}{lr}
\hline Patient room & $79(30)$ \\
\hline Operating room/ Recovery room & $51(19)$ \\
\hline Emergency department & $49(19)$ \\
\hline Outside patient room & $17(6)$ \\
\hline Service/ Utility room & $16(6)$ \\
\hline Outpatient clinic/ Office & $13(5)$ \\
\hline Others & $37(14)$
\end{tabular}

Was the injured worker the original user of the sharp item?

\begin{tabular}{lr}
\hline Yes & $201(77)$ \\
\hline No & $55(21)$ \\
\hline Unknown & $5(2)$ \\
\hline Source patient known & \\
\hline Yes & $191(73)$ \\
\hline No & $51(19)$ \\
\hline Unknown & $19(7)$ \\
\hline Not applicable & $1(0)$ \\
\hline
\end{tabular}


Table II. Device and injuries related characteristics, 2016-2018, Al Ahsa, Saudi Arabia ( $n=262$ )

Characteristic

Number (\%)

Was the sharp item contaminated?

Yes $223(85)$

No $17(6)$

Unknown $22(8)$

For what purpose was the sharp item originally used?

Injection, intra-muscular/subcutaneous, or other injection through the skin $58(22)$

To draw venous blood sample $39(15)$

Suturing $35(13)$

Unknown/Not applicable $26(10)$

To connect IV line (intermittent IV/piggyback/IV infusion/other IV line connection) $17(7)$

Cutting $13(5)$

To start IV or set up heparin lock (IV catheter or winged set-type needle) $10(4)$

Others $63(24)$

When did the injury occur?

During use of item (item slipped, patient moved, skin pinch-up, etc) $102(40)$

Between steps of a multi-step procedure (between incremental sorting, injections, passing $30(12)$ instruments, etc.)

After use, while recapping used needle $20(8)$

Other after use-before disposal (in transit to trash, cleaning, sorting, etc.) $20(8)$

After use, device left on floor, table, bed or inappropriate place $18(7)$

After use, while disassembling device or equipment $13(5)$

After disposal, item protruded from trash bag or inappropriate waste container $13(5)$

During disposal, while putting item into disposal container $11(4)$

Others $30(11)$

What type of device caused the injury?

Hollow-bore Needle $178(69)$

Surgical and solid needle $78(30)$

Glass 
Table III. Injury related characteristics, 2016-2018, Al Ahsa, Saudi Arabia (n=262)

Characteristic

Number (\%)

Location of the injury

Left hand $135(53)$

Right hand $115(45)$

Others

Type of injury

Superficial (little or no bleeding) $162(63)$

Moderate (skin punctured, some bleeding) $93(36)$

Severe (deep stick/cut, or profuse bleeding)

If injury was to a hand, did the sharp item penetrate?

Single pair of gloves

$194(75)$

Double pair of gloves $35(14)$

No gloves $26(10)$

Unknown

Dominant hand of the injured worker

Right-handed

Left-handed

$64(25)$

solid needles with $30 \%$. Among the hollow needle devices, disposable syringes (56\%) and other types of syringes (13\%) constituted the bulk of injuries. Surgical devices commonly involved in the injuries were suture needles (32\%), scalpels (17\%) and unspecified sharps (23\%). More than $85 \%$ of the sharps involved were reported to be contaminated.

Table III shows the injury related characteristics. It is evident that the dominant hand of the injured workers was the right hand mainly (75\% of HCWs) and the commonest location of injury was the left hand (53\% cases). More than $60 \%$ of the injuries were superficial with little or no bleeding. The sharps injuries were reported to have penetrated a single pair of gloves and double pair of gloves in $75 \%$ and $14 \%$ of cases respectively.

Almost all (93\%) of the injuries reported were with needle and sharp medical devices which were not of a "safety design". Only 4\% of devices had safety design and $3 \%$ reported as unknown. In the incident cases with safety design devices, the injury occurred "after activation" of the safety mechanism.

\section{Discussion}

As per our knowledge this is the first paper on sharps and needle stick injuries from Al Ahsa region, Saudi Arabia. There are many publications from other parts of Gulf and other parts of the world on this topic, but still there is lack of information about the regional variations and associated factors. Multiple aspects play a role in the occurrence and reporting of these injuries. A strong surveillance system is the foremost requirement to capture the incident injuries. Along with this, a robust system such as EPINet is needed to pick up the information correctly in a globally accepted format. There are challenges in using EPINet on ground with respect to capturing information on work experience, age and gender which are important 
information for planning interventions to reduce the injuries. Missing data is a major challenge which can hugely influence the quality of data collected. ${ }^{5}$

In our study the findings on the most at-risk job category and the type of device involved are similar to the figures in EPINet ${ }^{6}$ and many other studies. ${ }^{7-10}$ The data have been meticulously validated by a dedicated staff with constant trouble shooting when needed. Compared to the other similar studies which focus on few variables, the strength of our study is the description of almost all variables with very few missing data. The issue of missing data is a reality with EPINet and this can have an impact on the interpretation of results. Most of the previous studies are based on practices in single tertiary care hospitals. The present study discusses about the findings in 12 hospitals which include a mix of hospitals from primary to tertiary and from general to specialized centers.

In contrast to other studies, ${ }^{6,11}$ in our study we found that the injuries among housekeeping staff was quite high ( $10 \%$ of the total injuries). This is again supported by the corroborative information that in more than quarter of the reported injuries the "source patient" was not known and almost all the injuries reported by the housekeeping staff occur during handling medical waste, where in it is difficult to identify the source patient.

It is worth noting that the highest number of injuries have occurred in the morning shift compared to evening and night. This is owing to the fact of the patient load and the management related activities or interventions which are done in the morning hours. Similarly, maximum injuries have taken place in inpatient rooms, operating rooms and the emergency department which again reflect on the fact of the type of work and the pressure under which the HCWs perform their duties. ${ }^{12,13}$ These are obvious but important findings which need to be considered in planning preventive activities such as training, counseling of staff, uniform work distribution, ergonomics and effective task management.

It is a worrying finding that more than two-thirds of the injuries involved syringes and hollow bore needles and more than $85 \%$ of the devices reported in the injuries were contaminated. This raises the potential for acquiring blood borne pathogens as the chances of transmission are more in case of hollow bore needles. More than $50 \%$ of the injuries have taken place during the procedure which syncs with the information pertaining to peak hours and busy areas of work.

There are a few limitations in this study. Firstly, as the information is based on self-reporting, there is a risk of under-reporting owing to the fear of disciplinary action or loss of job. Secondly, there are many entries labelled as "others". This category constitutes a significant proportion and is difficult to interpret. Thirdly, it was out of the scope of the current study to see the number of exposed HCWs who developed any blood borne diseases as a sequela of the injury. The software used to capture the data (EPINet) has many fields which are not mandatory and this leads to the issue of "Missing data" as the data entry personnel on ground have a tendency to fill only mandatory fields. This leads to valuable data loss and inappropriate conclusions. Though this issue was taken care by one of our authors by feedback to hospitals and ensuring data completion, it is not a practical solution in routine program implementation.

It was found that most of the injuries occurred during the months of May and December. This might probably be due to the lack of staff and over-burden on the existing staff during these months. The month of May coincides with the holy month of Ramadan when the availability of staff is low and working hours are different. ${ }^{14,15}$ Similarly during the month of December, many staff go on vacation leading to low staff availability for work. These are our assumptions which need to be explored further in order to reduce the risk of injuries which occur to the tune of $25 \%$ in total for these 2 months. In our study only $4 \%$ of the injuries were related to usage of safety engineered devices (SED) and more than $90 \%$ of injuries occurred using devices which were non-SED. This can be a potential area to explore the usage of SEDs to prevent injuries. But there is no clear consensus on this issue as there are differing opinions on the efficacy of SEDs, from significant differences to no difference in the rates of injuries among staff using SEDs and 
non-SEDs. The quality of training and executing safe practices at work are more practical and long-term solutions to prevent injuries. ${ }^{16-20}$

\section{Conclusion}

In conclusion, we found that the nursing staff in busy hours and high patient load areas are more prone for injuries. Housekeeping staff need special focus as most of the injuries in this group occur with medical devices which are contaminated but from unknown sources.

We recommend that there has to be regular monitoring and supervision to ensure correct and complete data entry into the EPINet surveillance system. This would include regular physical visits or remote support to the staff engaged in data entry at service delivery points, periodic update trainings, regular reporting, data analysis and feedback on improvement of performance. Necessary modifications in the data collection tool is needed to ensure that the "missing data" is minimal.

The usage of SEDs to prevent injuries can be explored, depending on the local policies and management decisions, keeping in mind the costs involved. Tailor made training using the Pareto's principle of focusing on high risk staff and high risk work areas and onsite support, taking into considerations the above risk factors, would definitely go a long way in reducing these possibly fatal but preventable injuries caused by sharps and needles.

\section{Acknowledgments}

We would like to thank all the staff involved in entering the data of needle stick and sharps injuries in the EPINet program in all the hospitals of the region.

\section{References:}

1. Rapiti E, Prüss-Üstün A, Hutin Y. Sharps injuries: assessing the burden of disease from sharps injuries to health-care workers at national and local levels. Environmental Burden of Disease Series No. 11. https://www.who.int/quantifying_ehimpacts/ publications/ebd11.pdf [Accessed on 21 March 2019]

2. Beltrami EM, Williams IT, Shapiro CN, Chamberland ME. Risk and management of blood-borne infections in health care workers. Clin Microbiol Rev 2000; 13(3): 385-407. https://doi. org/10.1128/CMR.13.3.385-407.2000
3. WHO Health Report 2002; https://www.who.int/ occupational_health/topics/needinjuries/en/ [Accessed on 3rd February 2019]

4. National Institute for Occupational Safety and Health. Preventing needle stick injuries in health care settings. 1999. https://www.cdc.gov/niosh/docs/2000-108/pdfs/2000108.pdf?id=10.26616/NIOSHPUB2000108 [Accessed on 3rd February 2019]

5. Hyun K. The prevention and handling of the missing data. Korean J Anesthesiol 2013; 64(5): 402-406. https://doi. org/10.4097/kjae.2013.64.5.402

6. International Safety Center. EPINet report for needlestick and sharp object injuries 2017. https://internationalsafetycenter. org/wp-content/uploads/2018/10/Official-2017NeedleSummary.pdf [Accessed on 21 March 2019]

7. Park S, Jeong I, Huh J, Yoon Y, Lee S, Choi C. Needlestick and sharps injuries in a tertiary hospital in the Republic of Korea. Am J Infect Control 2008; 36(6): 439-443. https://doi. org/10.1016/j.ajic.2007.07.011

8. Memish ZA, Almuneef $M$, Dillon J. Epidemiology of needlestick and sharps injuries in a tertiary care center in Saudi Arabia. Am J Infect Control 2002; 30(4): 234-241. https://doi.org/10.1067/mic.2002.118841

9. Saulat J. Epidemiology of needlestick injuries among health care workers in a secondary care hospital in Saudi Arabia. Ann Saudi Med 2005; 25(3): 233-238. https://doi. org/10.5144/0256-4947.2005.233

10. Abu-Gad HA, Al-Turki KA. Some epidemiological aspects of needle stick injuries among the hospital health care workers: Eastern Province, Saudi Arabia. Eur J Epidemiol 2001; 17(5): 401-407. https://doi.org/10.1023/A:1013793301772

11. Abdulmageed SS, Alabbassi F, Alradi M, et al. Assessment of occupational exposure to sharp injuries among health care workers in King Abdulaziz University Hospital. Int J Community Med Public Health 2018; 5(5): 1756-1761. https:// doi.org/10.18203/2394-6040.ijcmph20181434

12. d'Ettorre G. Job stress and needlestick injuries: which targets for organizational interventions? Occupational Medicine 2016; 66: 678-680. https://doi.org/10.1093/occmed/kqw110

13. Akbari J, Taheri MR, Khosravi N, Zamani S, Ghadami A. Work related stress and needlestick injuries (NIs): A study among Iranian nurses with/without NIs. Ergonomics Int J 2018; 2(6): 000184. https://doi.org/10.23880/eoij-16000184

14. Wilburn SQ, Eijkemans G. Preventing needlestick injuries among healthcare workers: A WHO-ICN Collaboration. International Journal of Occupational and Environmental Health 2004; 10(4): 451-456. https://doi. org/10.1179/oeh.2004.10.4.451

15. Memish ZA, Assiri AM, Eldalatony MM, Hathout HM, Alzoman $\mathrm{H}$, Undaya $\mathrm{M}$. Risk analysis of needle stick and sharp object injuries among health care workers in a tertiary care hospital (Saudi Arabia). Journal of epidemiology and global health 2013; 3: 123-129. https://doi.org/10.1016/j.jegh.2013.03.004

16. Ballout RA, Diab B, Harb AC, Tarabay R, Khamassi S, AkI EA. Use of safety-engineered devices by healthcare workers for intravenous and/or phlebotomy procedures in healthcare settings: a systematic review and meta-analysis. BMC Health Serv Res 2016; 16: 458. https://doi.org/10.1186/s12913016-1705-y 
17. Schuurmans J, Lutgens SP, Groen L, Schneeberger PM. Do safety engineered devices reduce needlestick injuries? J Hosp Infect 2018; 100(1): 99-104. https://doi.org/10.1016/j. jhin.2018.04.026

18. Reddy VK, Lavoie MC, Verbeek JH, Pahwa M. Devices for preventing percutaneous exposure injuries caused by needles in healthcare personnel. Cochrane Database Syst Rev 2017; 11: CD009740. https://doi.org/10.1002/14651858.CD009740. pub3
19. Fukuda H, Yamanaka N. Reducing needlestick injuries through safety-engineered devices: results of a Japanese multicentre study. J Hosp Infect 2016; 92(2): 147-153. https://doi. org/10.1016/j.jhin.2015.09.019

20. Tosini W, Ciotti C, Goyer F, et al. Needlestick injury rates according to different types of safety-engineered devices: results of a French multicenter study. Infect Control Hosp Epidemiol 2010; 31(4): 402-407. https://doi. org/10.1086/651301 